Revista Bioética

\title{
PESQUISA
}

\section{Aprendizagem da geração millennial na graduação médica}

Paula Tamoto ${ }^{1}$, Renan dos Santos Gati ${ }^{1}$, João Marcelo Rondina ${ }^{1}$, Sérgio Luís Aparecido Brienze ${ }^{1}$, Alba Regina de Abreu Lima ${ }^{1}$, Júlio César André

1. Faculdade de Medicina de São José do Rio Preto, São José do Rio Preto/SP, Brasil.

\section{Resumo}

A maioria dos atuais graduandos nasceu entre 1982 e 2000. Trata-se dos chamados "millennials", e essa geração espera que a educação integre a tecnologia. Com isso, este estudo, transversal e descritivo-exploratório, propõe-se a conhecer a relação de estudantes de medicina do ciclo básico com as tecnologias interativas da webas quais podem melhorar o ensino -, visando fornecer informações para implementá-las com mais eficiência no meio acadêmico. Os resultados evidenciam que os alunos utilizam ferramentas da internet, mas com pouca diversidade, sendo as plataformas mais usadas o Google Docs, Facebook, YouTube e Dropbox. O artigo conclui que é necessário promover o contato com a tecnologia na educação médica a fim de preparar os alunos para enfrentar futuros desafios profissionais.

Palavras-chave: Educação de graduação em medicina. Mídias sociais. Tecnologia da informação. Internet.

\section{Resumen}

\section{Aprendizaje de la generación millennial en la graduación médica}

La mayoría de los estudiantes de grado actuales nacieron entre 1982 y 2000 . Son los llamados "millenials", generación que espera que la educación integre la tecnología. Este estudio descriptivo transversal y exploratorio se propone comprender la relación de los estudiantes de medicina del ciclo básico con las tecnologías interactivas de la web -que pueden mejorar la docencia-, con el objetivo de brindar información para implementarlas de manera más eficiente en el entorno académico. Los resultados muestran que los estudiantes utilizan herramientas de Internet, pero con poca diversidad, siendo las plataformas más utilizadas Google Docs, Facebook, YouTube y Dropbox. El artículo concluye que es necesario promover el contacto con la tecnología en la educación médica con el fin de preparar a los estudiantes para enfrentar los desafíos profesionales futuros.

Palabras clave: Educación de pregrado en medicina. Medios de comunicación sociales. Tecnología de la información. Internet.

\section{Abstract}

\section{Learning of the millennial generation in medical schools}

Currently, most undergraduate students are individuals born between 1982 and 2000, the so-called millennials, a generation that expects the integration of technology in education. Thus, this cross-sectional and descriptiveexploratory study proposes to understand the relationship of medical students with web-based technologies, which can improve learning, to implement them more efficiently in the academic environment. The results show that undergraduate medical students use these technologies, but with little diversity in services, being the most common Google Docs, Facebook, YouTube and Dropbox. We emphasize the need to expose students to technologies in medical education to overcome technological challenges faced by future physicians.

Keywords: Education, medical, undergraduate. Social media. Information technology. Internet. 
Atualmente, a maioria dos alunos de graduação, incluindo de medicina, nasceu entre 1982 e 2000, pertencendo portanto à geração dos chamados "millennials". Esse termo foi usado pela primeira vez por Strauss e Howe ${ }^{1}$, em 1992, no livro Generations: the history of America's future, 1584 to 2069. Desde então, a literatura sobre como esses indivíduos se comportam, interagem e preferem aprender vem crescendo, $\mathrm{e}$ professores de gerações anteriores lutam para entender os millennials e interagir com eles².

Na medicina, percebe-se que os estudantes millennials necessitam de mais feedback, mais interação com colegas e mais relações que envolvam sentimentos $^{3}$. De modo geral, os jovens dessa geração são mais assertivos, apresentam traços narcísicos e têm altas expectativas ${ }^{4}$. Dadas suas características únicas de personalidade, os millennials têm preferências, motivações e expectativas diferentes das gerações anteriores quanto a educação e formas de avaliação ${ }^{5}$.

Os millennials querem participar de experiências educacionais singulares, adaptadas a suas necessidades, em processo que foi denominado "napsterism" ${ }^{4}$ em referência a uma das primeiras plataformas de download de músicas, que permitia aos usuários criar listas de reprodução personalizadas. Esses alunos preferem a aprendizagem prática ${ }^{6}$ à leitura de textos longos ${ }^{4}$ e esperam que os professores observem suas habilidades em ambiente real ${ }^{2}$.

A integração da tecnologia na educação médica é esperada pelos alunos da geração $Y$ - outra denominação para os millennials -, visto que um a cada cinco desses jovens já estava acostumado a usar computadores quando completou 5 anos de idade, e o restante no máximo até os 18 anos $^{7}$. Para eles, a competência tecnológica significa relevância e capacidade de se relacionar com pessoas da mesma geração ${ }^{8}$, e muitos millennials estão ansiosos para usar contas de mídia social para fins educacionais ${ }^{9}$. Assim, percebe-se que as redes sociais estão integradas à vida cotidiana desse grupo, o que leva a separação menos definida entre trabalho e vida pessoal do que a observada nas gerações anteriores.

Tecnologias avançadas facilitam o aprendizado ao atender necessidades e oferecer aos alunos oportunidades de estudo, além de permitir que estudantes e professores compartilhem informações valiosas e acessem recursos independentemente da localização geográfica ${ }^{10}$. Na medicina, simuladores virtuais de pacientes, por exemplo, permitem praticar o diagnóstico sem risco de danos e observar patologias que não seriam prontamente disponíveis em encontros com pacientes reais ${ }^{11}$. Sistemas personalizados de realidade aumentada também podem promover o aprendizado autônomo, reduzindo a necessidade de materiais de laboratório e custos com instrutores ${ }^{12}$. Tais recursos, que incorporam ou sobrepõem as informações à realidade, despertam mais interesse do que os livros didáticos ${ }^{11,12}$.

As tecnologias interativas no ensino se baseiam nos recentes avanços computacionais. Todo software ou site que desencadeia novas ações em rede quando utilizado pode ser considerado interativo. Por exemplo, quando um usuário envia uma mensagem em mídia social, desencadeia-se ali uma interação com toda a rede. Isso acontece também em sites de URL compartilhada, blogs, wikis, plataformas de compartilhamento de filmes etc. Essas ferramentas muitas vezes reúnem funcionalidades e capacidades suficientes para que todas as interações sejam realizadas simultaneamente, na web, e se complementem entre $\mathrm{si}^{13}$.

Esse tipo de tecnologia tem criado novos espaços de construção de conhecimento e novas estratégias de ensino e aprendizagem ${ }^{14}$, ampliando o tempo de estudo. $O$ uso da tecnologia na sala de aula alimenta muitas discussões. Nesse novo modelo, as mídias digitais se incumbem de transmitir a informação propriamente dita, enquanto o professor alimenta discussões e instiga o pensamento crítico em casos clínicos e simulações. Estabelecimentos de ensino se interessam por essas novidades em parte porque as tecnologias interativas da web (TIW) são facilmente criadas, contam com grande adesão e flexibilizam os horários de estudo, permitindo aos alunos estabelecer seu próprio ritmo de aprendizagem ${ }^{15}$.

As TIW não se inserem somente nos métodos ativos do ensino médico. Ainda em métodos tradicionais, muitos professores estimulam estudantes a criar conteúdo, produzir e manipular imagens de vídeo (disponibilizando-as depois em serviços como o YouTube), usar palavras-chave (tags) para criar taxonomias que tornam a procura de informação em blogs mais eficaz ou a participar coletivamente da construção de enciclopédias virtuais como a Wikipédia ${ }^{16}$. Na medicina, há programas como o Homem Virtual, software de animação desenvolvido na Universidade de São Paulo para auxiliar o ensino de conteúdos de embriologia ou anatomia típicos do ciclo básico ${ }^{17}$.

Embora as TIW possam melhorar o ensino médico, esgotado pelo método tradicional ${ }^{18}$, os estudos que sustentam seu uso nesse contexto ainda são incipientes. Desconhece-se, inclusive, como essas tecnologias são efetivamente usadas pelos graduandos em medicina. Visando preencher parte dessa lacuna, este trabalho busca conhecer a relação de estudantes com a tecnologia com o propósito de fornecer informações para que as TIW sejam implementadas com mais eficiência no meio acadêmico. 
Método

Trata-se de estudo transversal, descritivo-exploratório. Os dados foram coletados na Faculdade de Medicina de São José do Rio Preto (Famerp), no interior do estado de São Paulo, que oferece cursos de enfermagem, medicina e psicologia. A amostra de conveniência foi composta por 113 acadêmicos do curso de medicina, de ambos os sexos, todos maiores de 18 anos. $O$ instrumento de coleta de dados foi aplicado em sala de aula, na data da avaliação final de disciplinas das duas primeiras séries, quando quase todos os estudantes estariam presentes.

O pesquisador apresentou o estudo, esclarecendo os participantes quanto ao tema e aos objetivos da pesquisa, informando ainda sobre a não obrigatoriedade de participação, o anonimato e demais aspectos éticos. Após a leitura e assinatura do termo de consentimento livre e esclarecido, o instrumento de coleta de dados foi aplicado. Para evitar contaminação da amostra, adotou-se a estratégia de impedir que os alunos conversassem sobre temas relativos ao instrumento enquanto o preenchessem. Para isso, os estudantes foram mantidos no mesmo ambiente em que se encontravam, de avaliação individual.

$\mathrm{O}$ instrumento de coleta de dados foi questionário estruturado que solicitava aos estudantes que escolhessem entre alternativas a fim de representar a frequência com que costumam utilizar uma série de TIW. A primeira parte do questionário continha dados gerais de caráter sociodemográfico. A segunda parte continha perguntas específicas, baseadas no prisma de conversação, e um mapa dinâmico sobre as principais redes sociais ${ }^{19}$. As TIW mais significantes e os objetos da pesquisa foram: ferramentas da própria instituição de ensino, jornalismo colaborativo, perguntas e respostas, colaboração, blogs, curadoria digital, redes de aprendizagem, fóruns de discussão, redes sociais, redes de negócios (business networking), vídeos, documentos e conteúdos, wikis, fotos e armazenamento em nuvem.
A análise exploratória dos dados incluiu média, mediana e desvio-padrão, bem como variação, para variáveis numéricas, e número e proporção, para variáveis categóricas. As variáveis ordinais em escala Likert foram representadas em média \pm desvio-padrão. Variáveis ordinais entre dois grupos não relacionados foram comparadas pelo teste de Mann-Whitney. Variáveis categóricas entre dois grupos não relacionados foram comparadas pelo teste qui-quadrado de Pearson ou exato de Fisher, quando apropriado. A análise estatística foi realizada no software IBM-SPSS Statistics versão 24 . Todos os testes foram bicaudais, e valores de $p<0,05$ foram considerados significantes.

\section{Resultados}

Foram incluídos no estudo 113 alunos, 52 (46\%) do primeiro ano do curso de medicina, $39(34,5 \%)$ do sexo masculino e $74(65,5 \%)$ do sexo feminino. Quarenta e seis $(40,7 \%)$ estudantes tinham 20 anos de idade ou menos, e 57 (50,4\%) tinham de 21 a 24 anos. Cem (88,5\%) participantes eram originários do estado de São Paulo, 11 (9,7\%) das demais unidades da federação, e outros $2(1,8 \%)$ não informaram esse dado. Noventa e um $(80,5 \%)$ vinham de áreas metropolitanas, e 13 (11,5\%) de áreas rurais.

Quanto ao uso de ferramentas impressas ou digitais, a maioria dos alunos afirmou usar livros físi$\cos (85 \%)$, resumos $(75,2 \%)$ e internet $(77 \%)$ para estudar, enquanto, em geral, e-books (62,8\%) e artigos $(55,8 \%)$ não foram utilizados para esse fim. Conforme a Tabela 1, número considerável de participantes concorda parcial ou plenamente que a internet melhora a aprendizagem $(93,8 \%)$, possibilita maior interação entre alunos e professor $(72,6 \%)$ e deve ser usada na sala de aula com orientação do docente $(66,4 \%)$. Todos concordam que a internet amplia as possibilidades de explorar o conteúdo, mas não há consenso sobre se ela aumenta a motivação para estudar (49,6\% acreditam que sim, $46 \%$ discordam).

Tabela 1. Opinião sobre o papel da internet na educação (São José do Rio Preto/SP, 2017)

\begin{tabular}{l|c|c|c|c|c} 
& $\begin{array}{c}\text { Concordo } \\
\text { plenamente } \\
\mathbf{n}(\%)\end{array}$ & $\begin{array}{c}\text { Concordo } \\
\mathbf{n}(\%)\end{array}$ & $\begin{array}{c}\text { Sem opinião } \\
\mathbf{n}(\%)\end{array}$ & $\begin{array}{c}\text { Discordo } \\
\mathbf{n}(\%)\end{array}$ & $\begin{array}{c}\text { Discordo } \\
\text { plenamente } \\
\mathbf{n}(\%)\end{array}$ \\
\hline Melhora a aprendizagem & $50(44,2)$ & $56(49,6)$ & $2(1,8)$ & $5(4,4)$ & $0(0,0)$ \\
\hline Motiva a estudar & $15(13,3)$ & $41(36,3)$ & $5(4,4)$ & $45(39,8)$ & $7(6,2)$ \\
\hline $\begin{array}{l}\text { Possibilita maior interação entre } \\
\text { alunos e professor }\end{array}$ & $25(22,1)$ & $57(50,4)$ & $8(7,1)$ & $22(19,5)$ & $1(0,9)$ \\
\hline $\begin{array}{l}\text { Amplia as possibilidades de } \\
\text { exploração do conteúdo }\end{array}$ & $66(58,4)$ & $47(41,6)$ & $0(0,0)$ & $0(0,0)$ & $0(0,0)$ \\
\hline $\begin{array}{l}\text { Deveria ser usada nas salas de } \\
\text { aula, com orientação do professor }\end{array}$ & $20(17,7)$ & $55(48,7)$ & $12(10,6)$ & $26(23,0)$ & $0(0,0)$ \\
\hline
\end{tabular}


De acordo com a Tabela 2, 59,3\% dos estudantes usam internet em casa para estudar, e $36,3 \%$ não utilizam rede sem fio. A maioria usa redes sociais $(93,8 \%)$, grupo no Facebook $(84,1 \%)$ e e-mail $(80,5 \%)$ para estudar - esses dois últimos criados especificamente para interação da turma do curso. A maior parte $(65,5 \%)$ também diz estar atualizada quanto aos tópicos formais e informais de conversação da turma na internet.

Tabela 2. Uso da internet e outras ferramentas para estudo (São José do Rio Preto/SP, 2017)

\begin{tabular}{l|c}
\multicolumn{1}{c|}{ Variável } & $\mathbf{n}(\%)$ \\
\hline Locais onde usa a internet para estudar* & \\
\hline Biblioteca da Famerp & $97(85,8)$ \\
\hline Casa & $67(59,3)$ \\
\hline Outras dependências da Famerp & $16(14,2)$ \\
\hline Outros lugares & $2(1,8)$ \\
\hline Uso do wi-fi da Famerp por semana & \\
\hline Não utiliza rede sem fio & $41(36,3)$ \\
\hline 1 dia por semana & $12(10,6)$ \\
\hline 3 ou 4 dias por semana & $28(24,8)$ \\
\hline Todos os dias & $29(25,7)$ \\
\hline Não respondeu & $3(2,6)$ \\
\hline Uso de rede social para estudar & \\
\hline Sim & $106(93,8)$ \\
\hline Não & $7(6,2)$ \\
\hline
\end{tabular}

continua..
Tabela 2. Continuação

\begin{tabular}{l|c}
\multicolumn{1}{c|}{ Variável } & $\mathbf{n ( \% )}$ \\
\hline Compra de softwares didáticos & $66(58,4)$ \\
\hline Nunca & $19(16,8)$ \\
\hline Raramente & $7(6,2)$ \\
\hline Às vezes & $1(0,9)$ \\
\hline Regularmente & $20(17,7)$ \\
\hline Não respondeu & \\
\hline Ferramentas utilizadas pela turma* & $95(84,1)$ \\
\hline Grupo no Facebook & $91(80,5)$ \\
\hline E-mail da sala & $18(15,9)$ \\
\hline Não respondeu & \\
\hline $\begin{array}{l}\text { Controle de tópicos formais e informais } \\
\text { de conversação da turma na internet }\end{array}$ & \\
\hline Sim & $74(65,5)$ \\
\hline Não & $21(18,6)$ \\
\hline Não respondeu & $18(15,9)$ \\
\hline Erapossve esolhermais deuma
\end{tabular}

*Era possivel escolher mais de uma alternativa nessas variáveis

A Tabela 3 mostra a frequência do uso de TIW pelos alunos. Das 14 categorias estudadas, os estudantes parecem ter contato significativo (mais de $40 \%$ de respostas "regularmente" e "sempre" em todas as subcategorias) com duas delas: "ferramentas da Famerp" e "redes sociais". Nas categorias "colaboração", "vídeos" e "armazenamento em nuvem", apenas Google Docs $(45,1 \%)$, YouTube $(81,4 \%)$ e Dropbox $(84,1 \%)$ apresentaram valores significativos. As demais subcategorias ficaram abaixo dos $40 \%$.

Tabela 3. Uso de tecnologias interativas da web (São José do Rio Preto/SP, 2017)

\begin{tabular}{|c|c|c|c|c|c|c|c|}
\hline & $\begin{array}{c}\text { Nunca } \\
\mathrm{n}(\%)\end{array}$ & $\begin{array}{c}\text { Raramente } \\
\text { n (\%) }\end{array}$ & $\begin{array}{c}\text { Às vezes } \\
n(\%)\end{array}$ & $\begin{array}{c}\text { Regularmente } \\
n(\%)\end{array}$ & $\begin{array}{c}\text { Sempre } \\
\text { n (\%) }\end{array}$ & $\begin{array}{c}\text { Não conhece } \\
\mathbf{n}(\%)\end{array}$ & $\begin{array}{c}\text { Não respondeu } \\
n(\%)\end{array}$ \\
\hline \multicolumn{8}{|l|}{ Ferramentas da Famerp } \\
\hline $\begin{array}{l}\text { Sistema de Gestão } \\
\text { Famerp, para } \\
\text { frequência, notas etc. }\end{array}$ & $1(0,9)$ & $5(4,4)$ & $15(13,3)$ & $30(26,5)$ & $61(54,0)$ & $0(0,0)$ & $1(0,9)$ \\
\hline $\begin{array}{l}\text { Sophia (plataforma } \\
\text { da biblioteca) }\end{array}$ & $17(15,0)$ & $5(4,4)$ & $19(16,8)$ & $33(29,2)$ & $38(33,6)$ & $0(0,0)$ & $1(0,9)$ \\
\hline \multicolumn{8}{|c|}{ Jornalismo colaborativo } \\
\hline Digg & $103(91,2)$ & $2(1,8)$ & $1(0,9)$ & $0(0,0)$ & $0(0,0)$ & $5(4,4)$ & $2(1,8)$ \\
\hline Reddit & $104(92,0)$ & $1(0,9)$ & $0(0,0)$ & $0(0,0)$ & $1(0,9)$ & $5(4,4)$ & $2(1,8)$ \\
\hline \multicolumn{8}{|l|}{ Perguntas e respostas } \\
\hline Yahoo & $52(46,0)$ & $20(17,7)$ & $25(22,1)$ & $9(8,0)$ & $5(4,4)$ & $1(0,9)$ & $1(0,9)$ \\
\hline Answers & $103(91,2)$ & $3(2,7)$ & $2(1,8)$ & $1(0,9)$ & $0(0,0)$ & $3(2,7)$ & $1(0,9)$ \\
\hline AllExperts & $105(92,9)$ & $2(1,8)$ & $2(1,8)$ & $0(0,0)$ & $0(0,0)$ & $3(2,7)$ & $1(0,9)$ \\
\hline \multicolumn{8}{|l|}{ Colaboração } \\
\hline Google Docs & $24(21,2)$ & $15(13,3)$ & $23(20,4)$ & $27(23,9)$ & $24(21,2)$ & $0(0,0)$ & $0(0,0)$ \\
\hline Microsoft Office & $59(52,2)$ & $5(4,4)$ & $12(10,6)$ & $15(13,3)$ & $21(18,6)$ & $1(0,9)$ & $0(0,0)$ \\
\hline Zoho & $105(92,9)$ & $3(2,7)$ & $1(0,9)$ & $1(0,9)$ & $1(0,9)$ & $2(1,8)$ & $0(0,0)$ \\
\hline Mindjet & $106(93,8)$ & $3(2,7)$ & $0(0,0)$ & $1(0,9)$ & $0(0,0)$ & $3(2,7)$ & $0(0,0)$ \\
\hline
\end{tabular}


Tabela 3. Continuação

\begin{tabular}{|c|c|c|c|c|c|c|c|}
\hline & $\begin{array}{c}\text { Nunca } \\
\text { n (\%) }\end{array}$ & $\begin{array}{c}\text { Raramente } \\
\text { n (\%) }\end{array}$ & $\begin{array}{c}\text { Às vezes } \\
\text { n (\%) }\end{array}$ & $\begin{array}{c}\text { Regularmente } \\
\text { n (\%) }\end{array}$ & $\begin{array}{c}\text { Sempre } \\
\text { n (\%) }\end{array}$ & $\begin{array}{c}\text { Não conhece } \\
n(\%)\end{array}$ & $\begin{array}{c}\text { Não respondeu } \\
n(\%)\end{array}$ \\
\hline \multicolumn{8}{|l|}{ Blogs } \\
\hline Blogger & $80(70,8)$ & $15(13,3)$ & $7(6,2)$ & $2(1,8)$ & $5(4,4)$ & $4(3,5)$ & $0(0,0)$ \\
\hline Tumblr & $77(68,1)$ & $11(9,7)$ & $11(9,7)$ & $8(7,1)$ & $3(2,7)$ & $3(2,7)$ & $0(0,0)$ \\
\hline WordPress & $88(77,9)$ & $10(8,8)$ & $5(4,4)$ & $4(3,5)$ & $2(1,8)$ & $4(3,5)$ & $0(0,0)$ \\
\hline \multicolumn{8}{|l|}{ Curadoria digital } \\
\hline Pinterest & $97(85,8)$ & $7(6,2)$ & $2(1,8)$ & $0(0,0)$ & $2(1,8)$ & $4(3,5)$ & $1(0,9)$ \\
\hline Paper.li & $108(95,6)$ & $1(0,9)$ & $0(0,0)$ & $0(0,0)$ & $0(0,0)$ & $3(2,7)$ & $1(0,9)$ \\
\hline Flipboard & $101(89,4)$ & $4(3,5)$ & $5(4,4)$ & $0(0,0)$ & $0(0,0)$ & $3(2,7)$ & $0(0,0)$ \\
\hline \multicolumn{8}{|l|}{ Fóruns de discussão } \\
\hline Google Groups & $39(34,5)$ & $16(14,2)$ & $23(20,4)$ & $16(14,2)$ & $17(15,0)$ & $1(0,9)$ & $1(0,9)$ \\
\hline \multicolumn{8}{|l|}{ Redes sociais } \\
\hline Facebook & $0(0,0)$ & $1(0,9)$ & $6(5,3)$ & $17(15,0)$ & $89(78,8)$ & $0(0,0)$ & $0(0,0)$ \\
\hline \multicolumn{8}{|l|}{ Redes de empresas } \\
\hline Plaxo & $109(96,5)$ & $0(0,0)$ & $0(0,0)$ & $0(0,0)$ & $0(0,0)$ & $3(2,7)$ & $1(0,9)$ \\
\hline LinkedIn & $100(88,5)$ & $7(6,2)$ & $1(0,9)$ & $0(0,0)$ & $1(0,9)$ & $3(2,7)$ & $1(0,9)$ \\
\hline \multicolumn{8}{|l|}{ Vídeos } \\
\hline YouTube & $0(0,0)$ & $4(3,5)$ & $17(15,0)$ & $26(23,0)$ & $66(58,4)$ & $0(0,0)$ & $0(0,0)$ \\
\hline Vimeo & $77(68,1)$ & $8(7,1)$ & $9(8,0)$ & $9(8,0)$ & $8(7,1)$ & $2(1,8)$ & $0(0,0)$ \\
\hline TED & $86(76,1)$ & $8(7,1)$ & $6(5,3)$ & $6(5,3)$ & $5(4,4)$ & $2(1,8)$ & $0(0,0)$ \\
\hline Vevo & $57(50,4)$ & $12(10,6)$ & $10(8,8)$ & $10(8,8)$ & $24(21,2)$ & $0(0,0)$ & $0(0,0)$ \\
\hline \multicolumn{8}{|c|}{ Documentos/conteúdo } \\
\hline ThinkFree & $82(72,6)$ & $5(4,4)$ & $7(6,2)$ & $9(8,0)$ & $8(7,1)$ & $2(1,8)$ & $0(0,0)$ \\
\hline Scribd & $82(72,6)$ & $7(6,2)$ & $10(8,8)$ & $3(2,7)$ & $1(0,9)$ & $3(2,7)$ & $7(6,2)$ \\
\hline SlideShare & $76(67,3)$ & $9(8,0)$ & $12(10,6)$ & $5(4,4)$ & $3(2,7)$ & $2(1,8)$ & $6(5,3)$ \\
\hline Prezi & $65(57,5)$ & $16(14,2)$ & $20(17,7)$ & $3(2,7)$ & $2(1,8)$ & $1(0,9)$ & $6(5,3)$ \\
\hline \multicolumn{8}{|l|}{ Wikis } \\
\hline Wikispace & $98(86,7)$ & $5(4,4)$ & $0(0,0)$ & $0(0,0)$ & $0(0,0)$ & $4(3,5)$ & $6(5,3)$ \\
\hline TWiki & $99(87,6)$ & $4(3,5)$ & $0(0,0)$ & $0(0,0)$ & $0(0,0)$ & $4(3,5)$ & $6(5,3)$ \\
\hline Wikia & $95(84,1)$ & $5(4,4)$ & $1(0,9)$ & $2(1,8)$ & $0(0,0)$ & $4(3,5)$ & $6(5,3)$ \\
\hline \multicolumn{8}{|l|}{ Fotos } \\
\hline Flickr & $87(77,0)$ & $8(7,1)$ & $6(5,3)$ & $3(2,7)$ & $1(0,9)$ & $2(1,8)$ & $6(5,3)$ \\
\hline Photobucket & $95(84,1)$ & $4(3,5)$ & $3(2,7)$ & $1(0,9)$ & $1(0,9)$ & $3(2,7)$ & $6(5,3)$ \\
\hline Picasa & $70(61,9)$ & $9(8,0)$ & $15(13,3)$ & $8(7,1)$ & $3(2,7)$ & $1(0,9)$ & $7(6,2)$ \\
\hline Facebook Camera & $78(69,0)$ & $7(6,2)$ & $7(6,2)$ & $4(3,5)$ & $10(8,8)$ & $1(0,9)$ & $6(5,3)$ \\
\hline Instagram & $48(42,5)$ & $9(8,0)$ & $18(15,9)$ & $12(10,6)$ & $17(15,0)$ & $2(1,8)$ & $7(6,2)$ \\
\hline \multicolumn{8}{|c|}{ Armazenamento em nuvem } \\
\hline Dropbox & $2(1,8)$ & $1(0,9)$ & $9(8,0)$ & $16(14,2)$ & $79(69,9)$ & $0(0,0)$ & $6(5,3)$ \\
\hline OneDrive & $69(61,1)$ & $7(6,2)$ & $11(9,7)$ & $6(5,3)$ & $13(11,5)$ & $1(0,9)$ & $6(5,3)$ \\
\hline Google Drive & $72(63,7)$ & $5(4,4)$ & $8(7,1)$ & $8(7,1)$ & $11(9,7)$ & $3(2,7)$ & $6(5,3)$ \\
\hline Apple iCloud & $75(66,4)$ & $2(1,8)$ & $8(7,1)$ & $4(3,5)$ & $16(14,2)$ & $2(1,8)$ & $6(5,3)$ \\
\hline Amazon Cloud & $101(89,4)$ & $1(0,9)$ & $2(1,8)$ & $0(0,0)$ & $0(0,0)$ & $3(2,7)$ & $6(5,3)$ \\
\hline
\end{tabular}


Analisando a frequência de uso de ferramentas impressas ou digitais em comparação com outros dados, percebeu-se que alunos da segunda série $(p=0,049)$ usam e-books mais regularmente e parecem estar mais atualizados quanto aos tópicos formais e informais de conversação da turma na internet $(p=0,002)$. A resposta afirmativa à variável "internet deveria ser usada nas salas de aula, com orientação do professor" foi mais significativa entre o sexo feminino $(p=0,030)$ e alunos da segunda série $(p=0,031)$. A utilização da internet em casa, para estudar, também foi significativamente maior entre alunos da segunda série $(p=0,006)$, resultado que se repetiu na frequência de uso do wi-fi da Famerp $(p=0,023)$, que ainda predominou entre estudantes oriundos do estado de São Paulo $(p=0,015)$. Todas as demais comparações não foram significativas.

\section{Discussão}

O fato de a maioria dos estudantes entrevistados ter até 29 anos de idade $(92,9 \%)$ os coloca como pertencentes à geração millennial, que em breve será a força de trabalho predominante e hoje já representa quase a totalidade dos médicos residentes. A predominância do sexo feminino $(65,5 \%)$ está de acordo com dados do Conselho Federal de Medicina ${ }^{20}$.

Ainda que o uso de e-books venha se disseminando entre estudantes universitários, há fortes indícios de preferência pelo livro físico como recurso de aprendizagem ${ }^{21-25}$, a partir da percepção de que seria mais fácil se concentrar ao ler cópias impressas ${ }^{21}$. Os livros digitais foram adotados com entusiasmo por bibliotecas acadêmicas, uma vez que resultariam em uso mais eficiente de recursos, economia de espaço e compatibilidade com hábitos da geração millennial. No entanto, apesar dessas vantagens, incluindo ainda portabilidade, disponibilidade e funcionalidade para pesquisa, o sentimento em relação aos e-books não é totalmente positivo. Há frustrações quanto à complexidade de aquisição desses livros, restrições de copyright das editoras e falta de compatibilidade com dispositivos de leitura ${ }^{26}$. Soma-se a isso o fato de que muitos estudantes desconhecem que as bibliotecas que frequentam disponibilizam e-books 22 .

Já o fato de o uso de e-books ser mais comum entre estudantes da segunda série pode estar relacionado ao processo de adaptação. Diniz e Almeida ${ }^{27}$ verificaram que, especialmente no primeiro semestre, os relacionamentos interpessoais são mais importantes do que a gestão de responsabilidades, que aumentam apenas a partir do segundo semestre. A inserção social no início do curso permite ao estudante construir um sentido partilhado de suas experiências - positivas e negativas - , ajudando-o a desenvolver estratégias de adaptação à universidade ${ }^{28}$, incluindo aí a familiarização com recursos de aprendizado disponíveis, como o e-book.

Apesar do consenso em relação ao papel da internet na educação ${ }^{29-31}$ (com exceção da motivação para estudar) e do rápido desenvolvimento das TIW nos últimos 15 anos, essas tecnologias ainda são pouco utilizadas no ensino de medicina. Percebe-se que falta motivação dos professores e recursos para o uso mais eficaz de mídias baseadas na internet ${ }^{32}$. Esses fatores talvez expliquem a ausência de consenso neste estudo no quesito TIW e motivação para estudar.

A tendência dos estudantes da segunda série em concordar com a afirmação de que a internet deve ser usada em sala de aula com orientação docente reforça sua dependência do professor, trazida do ensino médio e dos cursos pré-vestibulares e mantida nos métodos tradicionais de ensino. A percepção de que o uso bem-sucedido de grupos de aprendizado no Facebook depende de conexões sociais e liderança acadêmica preexistentes, seja por meio de estudantes comprometidos ou professores orientadores ${ }^{33}$, também pode estar na raiz dessa necessidade de orientação docente.

$O$ uso não generalizado da internet para estudar em casa, bem como seu predomínio entre estudantes da segunda série, pode estar relacionado ao processo de adaptação inicial, em termos de estar adequadamente "instalado" na cidade-sede da universidade e ter condições de contratar serviços de internet ou dividir despesas. Nesse mesmo sentido, a maior utilização do wi-fi da Famerp e o maior controle de tópicos formais e informais de conversação da turma na internet também parece resultar da adaptação.

Quanto às ferramentas online da faculdade o Sistema de Gestão Famerp, para controle de frequência e notas, e a plataforma da biblioteca, Sophia -, cabe destacar que esses serviços são essenciais para que os alunos administrem seu desempenho acadêmico e usufruam da biblioteca, o que justifica seu alto grau de acesso. Os resultados estão de acordo com a literatura, que destaca a importância de que os alunos, especialmente nas primeiras semanas após o ingresso, sejam informados sobre o que a universidade oferece (obtenção de documentos, procedimentos de matrícula, uso do restaurante universitário, localização das unidades e serviços, normas da instituição etc. ${ }^{28}$. 
Os serviços da categoria "colaboração" permitem que várias pessoas trabalhem simultaneamente em determinada tarefa, abrangendo ferramentas básicas de edição de texto, planilhas e apresentações. São bastante utilizados por estudantes, pois permitem criar e modificar arquivos sem a necessidade de instalar programas. Neste estudo, dos serviços apresentados, Google Docs foi o mais utilizado, convergindo com os resultados de Ríos ${ }^{34}$, que destaca como a plataforma favorece práticas que desenvolvem habilidades dos alunos ao estimular tanto o trabalho independente como em grupo. A autora ressalta ainda, como benefícios do serviço, a possibilidade de comunicação síncrona e assíncrona - superando barreiras espaçotemporais - e a interação para tomada de decisões em conjunto. Essas características fortalecem a comunicação entre professores e alunos e facilitam a avaliação e o feedback ${ }^{34}$.

Com os serviços de armazenamento em nuvem, o usuário pode salvar online e, caso queira, disponibilizar arquivos e dados de toda natureza. Os estudantes os utilizam para compartilhar apresentações, seminários e resumos, entre outros materiais didáticos. Dos serviços listados, Dropbox foi o mais utilizado, corroborando o estudo de Meske e colaboradores ${ }^{35}$, realizado com mais de 3 mil participantes, que também indicou demanda muito alta por essa plataforma no ensino superior alemão. A elevada utilização do Dropbox por estudantes universitários também é relatada por Ashtari e Eydgahi ${ }^{36}$, embora o serviço, nesse estudo, tenha ficado na segunda posição, atrás do Google Drive. A utilização do armazenamento em nuvem é menor entre estudantes de medicina de países de baixa renda ${ }^{37}$.

Atualmente, é necessário que médicos dominem tecnologias e saibam aplicá-las na busca por evidências científicas atualizadas que fundamentem a tomada de decisão ${ }^{38,39}$. As tecnologias da informação e comunicação podem ajudar a construir conhecimento e promover a aprendizagem centrada no aluno, e pesquisas recomendam sua integração ao ensino ${ }^{40-45}$. Dentre essas tecnologias, pode-se citar inclusive o Facebook ${ }^{46}$, cujo frequente uso observado neste estudo (93,8\% da amostra) está em consonância com estudos recentes ${ }^{10}$.

Essa rede social é especialmente bem aceita como ambiente de aprendizado e ensino por estudantes de graduação em medicina. Os alunos usam grupos abertos ou fechados do Facebook para se preparar para exames, compartilhar material online, discutir casos clínicos, organizar sessões presenciais e trocar informações sobre estágios ${ }^{46,47}$. Também há relato de implementação bem-sucedida de um grupo para ajudar estudantes de graduação a lidar com situações estressantes no primeiro ano da faculdade ${ }^{48}$. No entanto, apesar da boa aceitação do Facebook por grande parte dos discentes de medicina, não há evidências conclusivas sobre seu impacto como ambiente pessoal de aprendizado e ensino em níveis mais altos de competência clínica e resultados com pacientes ${ }^{46}$.

Sem dúvida, atualmente se vive fase de transformação educacional, buscando-se garantir que os modelos de treinamento, nos níveis de graduação, pós-graduação e educação continuada, produzam médicos que possam prosperar em ambientes desafiadores. No entanto, muitos estudantes de medicina da geração millennial ainda não exploraram completamente os benefícios das TIW para o aprendizado. Este estudo mostra isso quando constata que, em muitas das categorias pesquisadas ( 9 de 14), o contato com os serviços é ainda incipiente, mesmo no caso de plataformas cujo potencial como ferramenta de aprendizado já foi bastante explorado, como blogs ${ }^{44,49}$ e Twitter ${ }^{50,51}$ (este último não pesquisado aqui).

Nesse sentido, estudo relatou que estudantes de graduação podem se opor ao envolvimento formal do corpo docente no contexto informal do Facebook $^{33}$ e, segundo outra pesquisa, quando perguntados se aceitariam participar de cursos formais oferecidos por professores nessa rede social, apenas $30 \%$ responderam afirmativamente ${ }^{52}$. Já o YouTube, correntemente usado pelos estudantes da amostra, costuma ser apontado pela literatura como ferramenta de pouco valor educacional (contrariando as preferências da geração millennial) devido à natureza não supervisionada do conteúdo adicionado diariamente à plataforma ${ }^{45}$.

A pouca diversidade no uso das TIW já foi apontada ${ }^{53}$, e estudos mostram que alunos mencionam a falta de tempo e de conhecimento como obstáculos para explorar essas tecnologias ${ }^{36,37}$. A maioria gostaria de receber algum tipo de treinamento para utilizá-las ${ }^{37}$, o que mostra a importância de que a educação em TIW seja contemplada em todo o currículo médico, e não apenas nos primeiros anos de curso, como acontece na instituição pesquisada. Por fim, destaca-se ainda que é comum que alunos negligenciem aulas de TIW durante o primeiro e segundo anos da graduação devido à grande carga de trabalho de outras disciplinas, ignorando a importância do domínio da tecnologia no desenvolvimento profissional contínuo. Esse fato foi observado informalmente por todos os autores deste trabalho enquanto docentes, discentes e ex-discentes do curso de medicina da instituição pesquisada. 


\section{Considerações finais}

Os resultados evidenciam que os estudantes de medicina do ciclo básico que responderam ao questionário utilizam as TIW, mas com pouca diversidade. As tecnologias mais usadas, além das ferramentas da própria faculdade, foram Google Docs, Facebook, YouTube e Dropbox. Essa falta de diversidade remete a certo paradoxo, já que se esperava uso mais aprofundado das TIW por alunos da geração millennial.

Desenvolver estratégias de aprendizado apoiadas em TIW requer preparação mínima, tanto por parte do docente quanto dos estudantes. Assim, é necessário implementar políticas para fortalecer o domínio das tecnologias pelo estudante, considerando que a realidade social exigirá cada vez mais esse tipo de competência. Parte da responsabilidade pelos resultados deste estudo pode ser atribuída ao modelo tradicional de ensino adotado pelo curso e pelos próprios docentes, mas também é preciso que as universidades contem com conectividade e equipamentos adequados.
Quanto às limitações do estudo, há de se considerar que a resposta ao instrumento de coleta de dados pode ter certo viés, já que alguns participantes podem não se lembrar ou simplesmente não registrar corretamente o quanto acessam cada serviço. Ressalta-se também que, como é comum na internet, os serviços tendem a se valorizar ou desvalorizar constantemente, caindo em desuso ou ganhando popularidade rapidamente. No entanto, ainda que a preferência por determinada plataforma mude, o tipo de serviço procurado pelos estudantes continua a ser aproximadamente o mesmo, dada a importância dessas atividades desenvolvidas na internet.

Entender o uso das TIW é essencial para ajudar estudantes a enfrentar dilemas associados ao trabaIho em saúde no século XXI. A exposição a essas tecnologias durante a formação é extremamente importante para os futuros médicos. Por fim, ressalta-se a necessidade de repetir este estudo em momento posterior ao da atual pandemia da covid-19, que afetou os sistemas educacionais em todo o mundo, incluindo o ensino médico, ao exigir a passagem repentina para o chamado "ensino remoto emergencial".

\section{Referências}

1. Strauss W, Howe N. Generations: the history of America's future, 1584 to 2069. New York: William Morrow; 1992.

2. Roberts $D H$, Newman $L R$, Schwartzstein RM. Twelve tips for facilitating Millennials' learning. Med Teach [Internet]. 2012 [acesso 3 jun 2020];34(4):274-8. DOI: 10.3109/0142159X.2011.613498

3. Borges NJ, Manuel RS, Elam CL, Jones BJ. Differences in motives between Millennial and Generation X medical students. Med Educ [Internet]. 2010 [acesso 3 jun 2020];44(6):570-6. DOI: $10.1111 / \mathrm{j} .1365-2923.2010 .03633 . x$

4. Twenge JM. Generational changes and their impact in the classroom: teaching Generation Me. Med Educ [Internet]. 2009 [acesso 3 jun 2020];43(5):398-405. DOI: 10.1111/j.1365-2923.2009.03310.x

5. Desy JR, Reed DA, Wolanskyj AP. Milestones and millennials: a perfect pairing: competency-based medical education and the learning preferences of Generation Y. Mayo Clin Proc [Internet]. 2017 [acesso 3 jun 2020];92(2):243-50. DOI: 10.1016/j.mayocp.2016.10.026

6. Dede C. Planning for neomillennial learning styles. Educause Q [Internet]. 2005 [acesso 3 jun 2020];28(1):7-12. Disponível: https://bit.ly/34cfwkL

7. Kurup V. The new learners: millennials!! Int Anesthesiol Clin [Internet]. 2010 [acesso 3 jun 2020];48(3):13-25. DOI: 10.1097/AIA.0b013e3181e5c1b4

8. Price $C$. Why don't my students think I'm groovy? The new " $R$ "s for engaging millennial learners [Internet]. 2010 [acesso 3 jun 2020]. Disponível: https://bit.ly/3kiUXsh

9. Barry DS, Marzouk F, Chulak-Oglu K, Bennett D, Tierney P, O'Keeffe GW. Anatomy education for the YouTube generation. Anat Sci Educ [Internet]. 2016 [acesso 3 jun 2020];9(1):90-6. DOI: 10.1002/ase.1550

10. Purim KSM, Tizzot ELA. Protagonismo dos estudantes de medicina no uso do Facebook na graduação. Rev Bras Educ Med [Internet]. 2019 [acesso 3 jun 2020];43(1):187-96. DOI: 10.1590/1981-52712015v43n1rb20180139

11. Johnson TR, Lyons R, Chuah JH, Kopper R, Lok BC, Cendan JC. Optimal learning in a virtual patient simulation of cranial nerve palsies: the interaction between social learning context and student aptitude. Med Teach [Internet]. 2013 [acesso 3 jun 2020];35(1):e876-84. DOI: 10.3109/0142159X.2012.714884

12. Ma M, Fallavollita P, Seelbach I, Von Der Heide AM, Euler E, Waschke J, Navab N. Personalized augmented reality for anatomy education. Clin Anat [Internet]. 2016 [acesso 3 jun 2020];29(4):446-53. DOI: 10.1002/ca.22675

13. Rondina JM, Braile DM. Plataforma computacional Famerp virtual [tese]. São José do Rio Preto: Faculdade de Medicina de São José do Rio Preto; 2012.

14. Emery MF. O impacto das novas tecnologias no processo de ensino e aprendizagem. Rede Mebox [Internet]. 24 mar 2009 [acesso 3 jun 2020]. Disponível: https://bit.ly/37q3K8o 
15. Prober CG, Heath C. Lecture halls without lectures: a proposal for medical education. N Engl J Med [Internet]. 2012 [acesso 3 jun 2020];366(18):1657-9. DOI: 10.1056/NEJMp1202451

16. Cain J, Policastri A. Using Facebook as an informal learning environment. Am J Pharm Educ [Internet]. 2011 [acesso 3 jun 2020];75(10):207. DOI: 10.5688/ajpe7510207

17. Projeto Homem Virtual [Internet]. São Paulo: Universidade de São Paulo; 2003 [acesso 18 nov 2020]. Disponível: http://homemvirtual.org.br

18. Gotardelo DR, Gaspar JC. Dilemas em um currículo. Rev Méd Minas Gerais [Internet]. 2013 [acesso 3 jun 2020];22(4):438-41. Disponivel: https://bit.ly/3dJUA7M

19. Solis B, Thomas J. Introducing the conversation Prism. Marketing Sensei [Internet]. 2009 [acesso 3 jun 2020]. Disponível: https://bit.ly/35k9l87

20. Conselho Federal de Medicina. Demografia médica no Brasil 2018 [Internet]. São Paulo: Departamento de Medicina Preventiva da Faculdade de Medicina da USP; 2018 [acesso 3 jun 2020]. Disponível: https://bit.ly/3dKNsll

21. Baron N. Words onscreen: the fate of reading. New York: Oxford University Press; 2015.

22. Fenerick GMP, Silva MR. Percepção de estudantes quanto ao uso do acervo de e-books de uma biblioteca universitária. Biblos [Internet]. 2015 [acesso 3 jun 2020];29(2):5-23. Disponível: https://bit.ly/3dMBJZZ

23. Raynard M. Understanding academic e-books through the diffusion of innovations theory as a basis for developing effective marketing and educational strategies. J Acad Librariansh [Internet]. 2017 [acesso 3 jun 2020];43(1):82-6. DOI: 10.1016/j.acalib.2016.08.011

24. Paneto GP, Lyrio MRN. Uso de e-books pelos discentes graduandos dos cursos de arquivologia e biblioteconomia da Universidade Federal do Espírito Santo (UFES) [monografia]. Vitória: Universidade Federal do Espírito Santo; 2018.

25. Pretti MVF, Nascimento LAL. Acesso, uso e apropriação de e-books por estudantes universitários [Internet]. In: Anais do XXVIII Congresso Brasileiro de Biblioteconomia e Documentação; 1-4 out 2019; Vitória. São Paulo: Febab; 2019 [acesso 3 jun 2020]. Disponível: https://bit.ly/3dJ2b6G

26. Casselden B, Pears R. Higher education student pathways to ebook usage and engagement, and understanding: highways and cul de sacs. J Librarianship Inf Sci [Internet]. 2020 [acesso 3 jun 2020];52(2):601-19. DOI: 10.1177/0961000619841429

27. Diniz AM, Almeida LS. Adaptação à universidade em estudantes de primeiro ano: estudo diacrónico da interacção entre o relacionamento com pares, o bem-estar pessoal e o equilíbrio emocional. Anál Psicol [Internet]. 2006 [acesso 3 jun 2020];1(24):29-38. Disponível: https://bit.ly/3m9287c

28. Teixeira MAP, Dias ACG, Wottrich SH, Oliveira AM. Adaptação à universidade em jovens calouros. Psicol Esc Educ [Internet]. 2008 [acesso 28 out 2020];12(1):185-202. DOI: 10.1590/ S1413-85572008000100013

29. Cook DA, Levinson AJ, Garside S, Dupras DM, Erwin PJ, Montori VM. Internet-based learning in the health professions: a meta-analysis. Jama [Internet]. 2008 [acesso 3 jun 2020];300(10):1181-96. DOI: 10.1001/jama.300.10.1181

30. Wong G, Greenhalgh T, Pawson R. Internet-based medical education: a realist review of what works, for whom and in what circumstances. BMC Med Educ [Internet]. 2010 [acesso 3 jun 2020];10:12. DOI: 10.1186/1472-6920-10-12

31. Sklar DP. Can words on the screen replace the face in the classroom? Using the internet to revolutionize medical education. Acad Med [Internet]. 2018 [acesso 3 jun 2020];93(8):1095-7. DOI: 10.1097/ACM.0000000000002272

32. Vogelsang M, Rockenbauch K, Wrigge H, Heinke W, Hempel G. Medical education for "Generation Z": everything online?! An analysis of internet-based media use by teachers in medicine. GMS J Medical Educ [Internet]. 2018 [acesso 3 jun 2020];35(2):Doc21. DOI: 10.3205/zma001168

33. George DR. "Friending Facebook?" A minicourse on the use of social media by health professionals. J Contin Educ Health Prof [Internet]. 2011 [acesso 3 jun 2020];31(3):215-9. DOI: 10.1002/chp.20129

34. Ríos M. Google Docs en educación universitaria: usos para la enseñanza y el aprendizaje en la licenciatura en educación de los EUS-UCV [Internet]. In: Anales de la XIV Jornada y V Congreso Internacional de Investigación Educativa; 17-21 out 2016; Caracas. Caracas: Centro de Investigaciones Educativas; 2016 [acesso 3 jun 2020]. Disponível: https://bit.ly/3ki4Kz6

35. Meske C, Stieglitz S, Vogl R, Rudolph D, Oksuz A. Cloud storage services in higher education: results of a preliminary study in the context of the Sync\&Share-Project in Germany [Internet]. In: Proceedings of the International Conference on Learning and Collaboration Technologies; 22-37 jun 2014; Heraklion. Cham: Springer; 2014 [acesso 3 jun 2020]. p. 161-71. DOI: 10.1007/978-3-319-07482-5_16

36. Ashtari S, Eydgahi A. Student perceptions of cloud applications effectiveness in higher education. J Comput Sci [Internet]. 2017 [acesso 3 jun 2020];23:173-80. DOI: 10.1016/j.jocs.2016.12.007

37. Hettige $S$, Dasanayaka E, Ediriweera DS. Usage of cloud storage facilities by medical students in a low-middle income country, Sri Lanka: a cross sectional study. BMC Medical Inform Decis Mak [Internet]. 2020 [acesso 3 jun 2020];20:10. DOI: 10.1186/s12911-020-1029-z

38. Brasil. Ministério da Educação. Resolução CNE/CES no 3, de 20 de junho de 2014. Institui diretrizes curriculares nacionais do curso de graduação em medicina e dá outras providências. Diário Oficial da União [Internet]. Brasília, p. 8-11, 23 jun 2014 [acesso 3 jun 2020]. Seção 1. Disponível: https://bit.ly/2HfPQLw

39. Simpkin AL, Walesby KE. Training tomorrow's doctors. Future Hosp J [Internet]. 2017 [acesso 3 jun 2020];4(1):56-60. Disponível: https://bit.ly/3dKB238 
40. Kind T, Patel PD, Lie D, Chretien KC. Twelve tips for using social media as a medical educator. Med Teach [Internet]. 2014 [acesso 3 jun 2020];36(4):284-90. DOI: 10.3109/0142159X.2013.852167

41. Silva JR, Medeiros FB, Moura FMS, Bessa WS, Bezerra ELM. Uso das tecnologias de informação e comunicação no curso de medicina da UFRN. Rev Bras Educ Med [Internet]. 2015 [acesso 3 jun 2020];39(4):537-41. DOI: 10.1590/1981-52712015v39n4e02562014

42. Pereira TA, Tarcia RML, Areco KCN, Sigulem D. Uso das tecnologias de informação e comunicação por professores da área da saúde da Universidade Federal de São Paulo. Rev Bras Educ Med [Internet]. 2016 [acesso 3 jun 2020];40(1):59-6. Disponível: https://bit.ly/34kc7jG

43. Galiatsatos P, Porto-Carreiro F, Hayashi J, Zakaria S, Christmas C. The use of social media to supplement resident medical education: the SMART-ME initiative. Med Educ Online [Internet]. 2016 [acesso 3 jun 2020];21(1):29332. DOI: 10.3402/meo.v21.29332

44. Sterling M, Leung P, Wright D, Bishop TF. The use of social media in graduate medical education: a systematic review. Acad Med [Internet]. 2017 [acesso 3 jun 2020];92(7):1043-56. DOI: 10.1097/ACM.0000000000001617

45. Sutherland S, Jalali A. Social media as an open-learning resource in medical education: current perspectives. Adv Med Educ Pract [Internet]. 2017 [acesso 3 jun 2020];8:369-75. DOI: 10.2147/AMEP.S112594

46. Pander T, Pinilla S, Dimitriadis K, Fischer MR. The use of Facebook in medical education: a literature review. GMS J Medical Educ [Internet]. 2014 [acesso 3 jun 2020];31(3):Doc33. DOI: 10.3205/zma000925

47. MacDonald J, Sohn S, Ellis P. Privacy, professionalism and Facebook: a dilemma for young doctors. Med Educ [Internet]. 2010 [acesso 3 jun 2020];44(8):805-13. DOI: 10.1111/j.13652923.2010.03720.x

48. Finn G, Garner J, Sawdon M. "You're judged all the time!" Students' views on professionalism: a multicentre study. Med Educ [Internet]. 2010 [acesso 3 jun 2020];44(8):814-25. DOI: 10.1111/j.1365-2923.2010.03743.x

49. Downes S. Educational blogging. Educause Rev [Internet]. 2004 [acesso 3 jun 2020];39(5):14-26. Disponível: https://bit.ly/2Hri7yu

50. Jalali A, Wood TJ. Tweeting during conferences: educational or just another distraction? Med Educ [Internet]. 2013 [acesso 3 jun 2020];47(11):1129-30. DOI: 10.1111/medu.12337

51. Jalali A, Sherbino J, Frank F, Sutherland S. Social media and medical education: exploring the potential of Twitter as a learning tool. Int Rev Psychiatry [Internet]. 2015 [acesso 3 jun 2020];27(2):140-6. DOI: 10.3109/09540261.2015.1015502

52. Garner J, O'Sullivan H. Facebook and the professional behaviours of undergraduate medical students. Clin Teach [Internet]. 2010 [acesso 3 jun 2020];7(2):112-5. DOI: 10.1111/j.1743-498X.2010.00356.x

53. Biscardi GT, Rondina JM. Padrões de uso da tecnologia digital no aprendizado de um curso de medicina. Rev Bras Inform Educ [Internet]. 2017 [acesso 3 jun 2020];25(1):1-15. DOI: 10.5753/RBIE.2017.25.01.1

\section{Participação dos autores}

Paula Tamoto e João Marcelo Rondina conceberam e desenharam o projeto, coletaram os dados e, com Renan dos Santos Gati, que contribuiu ainda com a elaboração do manuscrito, analisaram e interpretaram as informações. Júlio César André, Sérgio Luís Aparecido Brienze e Alba Regina de Abreu Lima redigiram o manuscrito e revisaram criticamente importantes conteúdos. Todos os autores leram e aprovaram a versão a ser publicada.

\section{Correspondência}

João Marcelo Rondina - Faculdade de Medicina de São José do Rio Preto. Av. Brigadeiro Faria Lima, 5.416, Vila São Pedro CEP 15090-000. São José do Rio Preto/SP, Brasil.

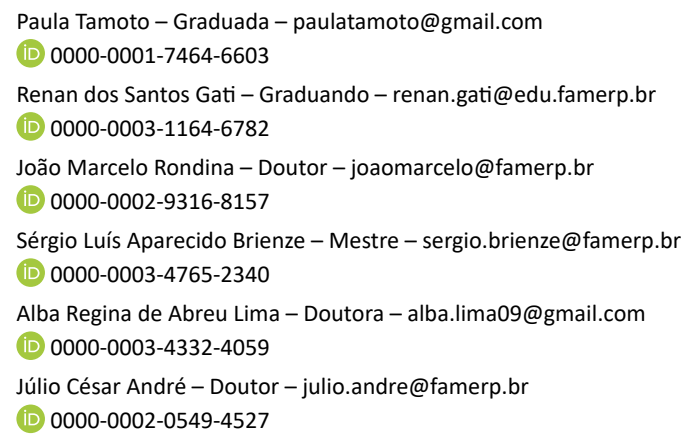

